Research Paper

\title{
Psychometric Properties of the Persian Version of Penn Parkinson Daily Activities Questionnaire-15
}

\author{
Negar Nikbakht ${ }^{1}$ (D), Mehdi Rezaee ${ }^{1 *}$ (D), Minoo Kalantari ${ }^{1}$, Seyed Mehdi Tabatabaee $^{2}$ (D), Gholam Ali Shahidi ${ }^{3}$ (i) \\ 1. Department of Occupational Therapy, School of Rehabilitation, Shahid Beheshti University of Medical Sciences, Tehran, Iran. \\ 2. Department of Basic Sciences, School of Rehabilitation, Shahid Beheshti University of Medical Sciences, Tehran, Iran. \\ 3. Department of Neurology, Iran University of Medical Sciences, Hazrat Rasool Hospital, Tehran, Iran.
}

\begin{tabular}{|c|c|}
\hline $\begin{array}{l}\text { Use your device to scan } \\
\text { and read the article online }\end{array}$ & ditation Nikbakht, N., Rezaee, M., Kalantari1, M., Tabatabaee, S. M., and Shahidi, G. A. (2022). Psychometric \\
\hline 口ifiris & $\begin{array}{l}\text { Properties of the Persian Version of Penn Parkinson Daily Activities Questionnaire-15. Basic and Clinical Neuroscience, } \\
\text { 13(5), 685-694. http://dx.doi.org/10.32598/bcn.2021.2410.1 }\end{array}$ \\
\hline rbstris & dol http://dx.doi.org/10.32598/ben.2021.2410.1 \\
\hline
\end{tabular}

\section{(i) (3)}

Article info:

Received: 17 Feb 2020

First Revision: 16 May 2020

Accepted: 28 Jun 2020

Available Online: $01 \mathrm{Sep} 2022$

Keywords:

Psychometric properties, Parkinson disease, Cognition, Instrumental activities of the daily living, Persian version

\begin{abstract}
A B S T RAC T
Introduction: Appropriate information about the ability of patients with Parkinson disease (PD) to perform cognitive instrumental activities of daily living (IADL) is necessary. The present study aimed to assess the psychometric properties of the Persian version of the Penn Parkinson daily activities questionnaire-15 (PDAQ-15).

Methods: A total of 165 knowledgeable informants of PD patients completed the PDAQ-15. The clinical dementia rating scale, Hoehn and Yahr staging, hospital anxiety and depression scale (HADS), and Lawton IADL scale were used in the study. Internal consistency and test-retest reliability were evaluated by the Cronbach $\alpha$ coefficient and intraclass correlation coefficient (ICC), respectively. To examine the dimensionality of the questionnaire, exploratory factor analysis was used. The construct validity was assessed using the Spearman rank correlation test. To assess the discriminative validity, PDAQ-15 scores were compared across cognitive stages.
\end{abstract}

Results: The PDAQ-15 showed strong internal consistency (the Cronbach $\alpha=0.99$ ) and test-retest reliability $(\mathrm{ICC}=0.99)$. Only one dimension was identified for the PDAQ15 in the factor analysis. There was a strong correlation between PDAQ-15 with the depression domain of the HADS scale and the Lawton IADL scale ( $\mathrm{rs}=|0.71-0.95|)$. The correlation of PDAQ-15 with the anxiety domain of the HADS scale was moderate $(\mathrm{rs}=0.66)$. Discriminative validity analysis showed that the PDAQ-15 has significant power to discriminate between PD patients across cognitive stages.

Conclusion: These results suggest that the PDAQ-15 is a valid and reliable PD-specific instrument and can be useful in clinical and research settings.

\section{* Corresponding Author:}

Mehdi Rezaee, PhD.

Address: Department of Occupational Therapy, School of Rehabilitation, Shahid Beheshti University of Medical Sciences, Tehran, Iran. Tel: +98 (912) 3848858

E-mail: arezaee2003@yahoo.com 


\section{Highlights}

- Cognitive impairments affect the ability to perform daily activities in patients with Parkinson disease (PD).

- The Penn Parkinson's daily activities questionnaire-15 (PDAQ-15) is a suitable tool to assess daily cognitive function in patients with Parkinson disease.

- The PDAQ-15 has discriminated well between patients with Parkinson disease across cognitive stages.

\section{Plain Language Summary}

Parkinson disease (PD) is one of the most common diseases in the elderly. Cognitive impairment is the main problem in PD that affects the patients' abilities to perform activities of daily living and leads to disability and suffering for their caregivers. Occupational therapists, as the key members of the rehabilitation team, evaluate and treat patients in their daily activities to enhance their independence. The tools for measuring daily activities play an important role in assessing the level of functional independence and promoting new cognitive therapies in these patients. Therefore, the present study aimed to prepare a Persian version of the Penn Parkinson's daily activities questionnaire-15 (PDAQ-15) questionnaire for people with PD in Iran. In this study, we evaluate the ability of patients with Parkinson disease with different cognitive levels. Our results showed that this questionnaire is a suitable tool to assess the cognitive and daily function of patients with PD. It is hoped that the results of this study help target and plan cognitive therapy strategies for PD.

\section{Introduction}

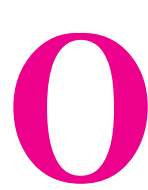

ne of the common and important non-motor symptoms of Parkinson disease (PD) is a cognitive impairment that causes disability, worsens quality of life (QoL), and increases patients' mortality and their caregivers' burden (Auclair-Ouellet et al., 2017; Leung et al., 2015). There is one classification for cognitive deficits in $\mathrm{PD}$, ranging from mild cognitive impairment (PD-MCI) to PD Dementia (PDD). Patients with PD-MCI are at great risk of developing PDD (Federico et al., 2017). Reports from the Movement Disorder Society indicate that $26.7 \%$ of PD patients are PD-MCI type and 30\% to $40 \%$ PDD (Dancis \& Cotter, 2015). The cognitive domains affected during PD include attention, memory, visuospatial abilities, and executive functions (Siciliano et al., 2017). These cognitive deficits impact instrumental activities of daily living (IADLs), such as driving, financial skills, and medication management, and PD patients with dementia have more functional limitations (Fernández-Bobadilla et al., 2017; Martin et al., 2013; Ruzafa-Valiente et al., 2016).

One of the therapeutic interventions in PD is the identification of cognitive impairments to improve the patients' functions (Brennan et al., 2016a). Therefore, an instrument to assess functional changes related to $\operatorname{cog}$ nitive impairment can improve clinical management and evaluation of new cognitive therapies in PD (Bren- nan et al., 2016a; Kulisevsky et al., 2013). Also, IADL measurement scales can improve rehabilitation services. Among the rehab professionals, occupational therapists are the key members who help PD patients with the restoration, maintenance, and promotion of participation in meaningful activities (Foster \& Hershey, 2011; Soltanmohamadi et al., 2014).

Several scales are used to measure IADLs but do not consider the specific features of PD, including cognitive impairments (Brennan et al., 2016a). Only two published functional scales have been specifically designed to assess IADL in PD: the Parkinson disease cognitive functional rating scale (PD-CFRS) and the Penn Parkinson's daily activities questionnaire-15 (PDAQ-15). The PD-CFRS is a PD-specific questionnaire that takes 5 minutes to complete and has 12 items sensitive to mild cognitive impairment that was administered to a knowledgeable informant (KI) in the interview. This scale provided appropriate validity and reliability in the initial validation study (Brennan et al., 2016b). The initial version of PDAQ was designed in 2015 in the United States by Laura Brennan et al. This tool uses the item-response theory (IRT) methodology to assess board range of cognitive IADL functions in PD. The average time required to complete the 50-item PDAQ is 10-15 min; therefore, the PDAQ-15 version was prepared as a brief instrument of the IADL function (Brennan et al., 2016b). 
The reasons for choosing the PDAQ-15 for psychometric properties analysis were its advantages over the PD-CFRS. For example, PDAQ-15 is derived from the original 50-item version, which used IRT in the developing process. Also, the strong psychometric properties of the original version in a large sample of patients is another reason for the superiority of the PDAQ-15 (Brennan et al., 2016b). Due to the lack of cognitive IADL scales in PD among the Iranian population, the present study aimed to measure the validity and reliability of the PDAQ-15.

\section{Materials and Methods}

\section{Study subjects}

Recruited PD patients $(\mathrm{n}=165)$ were a convenience sample of outpatients referred to a neurology clinic in the center of Tehran City, Iran, with the highest number of referrals from all over Iran. The inclusion criteria were as follows: standard criteria for idiopathic PD (Hughes, Daniel et al., 1992) based on the two neurologists' diagnosis; those who had an educated key informant (KI), such as a spouse, child, paid caregiver, or other individuals that have daily contact with the patient available to complete the PDAQ-15 being in levodopa on-state; absence of causes that were interfering with cognitive status (e.g., other neurological disorder, brain surgery, psychosis, medications, etc. ) by a brief semi-structured interview with $\mathrm{KI}$ and patient.

\section{Study procedure}

The translation and face and content validity of the PDAQ-15 has been described in detail (Nikbakht et al., 2018), and the following steps were performed for the present study. Initially, all required information, including demographic characteristics and clinical assessments such as age, education, gender, age of onset, disease duration, Levodopa equivalent daily doses (LEDD), Dopamine Agonist (DA), clinical dementia rating (CDR), Hoehn and Yahr (H\&Y), hospital anxiety and depression scale (HADS), PDAQ-15, and Lawton IADL scale were identified. Then, to examine convergent validity, PDAQ-15, and Lawton IADL were completed by a key informant (one person) for each participant. The Lawton IADL scale was scored based on functional deficit due to cognitive loss, not physical dysfunction. The only investigator involved in rating the CDR was blinded to the obtained scores by KIs in completing the PDAQ-15 and the Lawton IADL scale. To assess the test-retest reliability, the PDAQ-15 was completed by the same KI for the second time after two weeks. All assessments were conducted in one day while in the levodopa on-state. Evaluations were performed in a quiet room in the clinic, and patients were given sufficient time to complete the questionnaire.

\section{Assessments}

The PDAQ is a PD-specific questionnaire comprising 15 items completed by a KI of a PD patient. Each item contains a question about the level of difficulty in performing an IADL by a PD patient and was scored based on KI rating on the following scale: "none," "a little," "somewhat," "a lot," and "cannot do." Scores ranged from 0 to 4 for each item (total score range $=0-60$ ), with higher scores indicating better cognitive IADL function. The PDAQ-15 demonstrated strong psychometric properties across cognitive stages (Brennan et al., 2016b).

Lawton IADL scale is a valid and reliable instrument to assess the ability to perform more complex activities of daily living. The Lawton measures 8 domains of cooking, telephone use, shopping, financial management, housekeeping, doing laundry, using transportation, and handling medication, which scored from 0 to 8 . Low scores indicate low function or dependency, and high scores indicate high function or independence. This scale can be administered by either a questionnaire or in a 10 to 15 minutes interview. The answers can be provided by either the patient, KI, or caregiver. Each ability assessed by Lawton depends on physical and cognitive function. This scale can be scored in different ways, depending on the purpose of the evaluation and how the information is used (Graf, 2008; Lawton \& BRODY, 1970). The Lawton IADL scale has already been used in PD studies (Cahn et al., 1998; Christ et al., 2013; Rasovska \& Rektorova, 2011) and has shown good ability to be a valid and reliable instrument for the assessment of IADL in the Persian version (Soltanmohamadi et al., 2014).

The CDR is a valid and reliable scale to rate six domains associated with dementia: memory, orientation, judgment and problem-solving, community affairs, home and hobbies, and personal care. This scale uses a semi-structured interview with the subject and his/ her family. In rating each of these domains, the assessment should be on the patient's cognitive ability to function in these areas, not because of physical frailty. Although the CDR was developed primarily for use in patients with Alzheimer disease, adjusted CDR cut-off scores for patients with PD have provided and shown acceptable validity (Morris, 1997). The CDR classifies PD patients as having normal cognition (PD-NC) 
$(\mathrm{CDR}=0)$, PD-MCI $(\mathrm{CDR}=0.5)$, or $\mathrm{PDD}(\mathrm{CDR} \geq 1)$ (Wyman-Chick \& Scott, 2015).

The H\&Y is a widely used clinical rating scale. On this scale, 5 levels are defined to determine the severity of PD progression. For example, level 1 indicates normal status, and level 5 indicates the use of a wheelchair (Mehdizadeh et al., 2019).

The HADS is a brief and widely-used 14-item scale to measure current anxiety and depression in nonpsychiatric hospital patients. The HADS has independent subscales for anxiety and depression. Scores on each scale range as follows: normal (0-7), mild (810), moderate (11-14), and severe (15-21) (Zigmond \& Snaith, 1983). The Persian version of HADS has demonstrated high validity and reliability (Montazeri et al., 2003). Since anxiety and depression are two of the most important factors involved in the cognitive status of PD patients, they were evaluated in this study (Kulisevsky et al., 2013).

\section{Statistical analysis}

Descriptive statistics, including means, percentages, and standard deviations, were calculated for demographics and clinimetric characteristics. The Shapiro-Francia test was used to assess the data distribution, and the result showed that a total score of PDAQ-15 was not normally distributed. Acceptability was assessed, considering ceiling and floor effects at a level $\leq 15 \%$ (McHorney $\&$ Tarlov, 1995). The acceptable range for skewness is from -1 to +1 (Hays et al., 1993).

To assess the internal consistency of the PDAQ-15, the Cronbach alpha coefficient (with values $>0.70$ considered adequate) was calculated (Lohr, 2002). To investigate the relationship between items, the inter-item correlation method was measured, considering a correlation coefficient $\geq 0.2$ acceptable (Piedmont, 2014). The values $\geq 0.20$ were considered standard thresholds to determine the corrected item-total correlation (Streiner et al., 2015). The test-retest reliability was checked for total score using the intraclass correlation (ICC) coefficient, considering ICC above 0.70 as adequate reliability (Terwee et al., 2007).

The standard error of measurement (SEM) was calculated to assess the precision of the PDAQ- 15 . The variety of the score in repeating the measurement is determined by SEM. In the SEM=SD $\sqrt{ }(1-\mathrm{rxx})$ formula, $\mathrm{SD}$ is obtained from the first assessment total score, and rxx is the
ICC from the test-retest. The SEM $<1.2 \mathrm{SD}$ was considered acceptable in our study (Taghizadeh et al., 2018).

To check the dimensionality of the PDAQ-15, the exploratory factor analysis (principal component analysis) with varimax rotation (eigenvalues $\geq 1$ ) was carried out (Gorsuch, 1997). Convergent validity was assessed using the Spearman rank correlation test to examine the correlation between the total PDAQ-15 score with the Lawton IADL scale and HADS. Coefficient values $<0.30$ were considered weak, 0.30-0.70 moderate, and $>0.70$ strong correlation (Simon, 2006).

Discriminative validity analysis was assessed using the Kruskal-Wallis test and Cohen's d effect size to determine the ability of the PDAQ-5 to differentiate between PD cognitive stages. A Cohen's d effect size of $0.2,0.5$, and 0.8 indicate small, medium, and high magnitude of difference between stages, respectively (Husted et al., 2000).

\section{Results}

Table 1 presents the demographic information and clinical characteristics for all participants (165 PD patients) and by cognitive diagnosis based on CDR score (i.e., normal cognition, MCI, or dementia). The CDR results were $45.5 \%$ PD-NC $(\mathrm{n}=75), 30.3 \%$ PD-MCI $(\mathrm{n}=50)$, and $24.2 \% \mathrm{PDD}(\mathrm{n}=40)$.

The ceiling and floor effects for the total PDAQ-15 score were $18.2 \%$ and $7.9 \%$, respectively, and the skewness was -1.15 . The PDAQ-15 showed high internal consistency (the Cronbach alpha $=0.99$; no item improved the Cronbach alpha if removed). The inter-item correlation of the PDAQ-15 ranged from 0.90 to 0.99 . The corrected item-total correlations coefficient ranged from 0.94 for item 9 (How much difficulty does the patient currently have remembering new information like phone numbers or simple instructions?) to 0.98 for item 3 (How much difficulty does the patient currently have counting the correct amount of money when making purchases?) (Table 2). The ICC for the total PDAQ-15 score for test-retest was high $(0.99 ; 95 \% \mathrm{CI})$. The SEM of the PDAQ-15 was 1.89 (1/2 SD value=9.46).

Factor analysis for the PDAQ-15 with varimax rotation showed only one component (eigenvalue $=14.22$; total variance $=94.81$; Kaiser-Meyer-Olkin $=0.96$; Bartlett's sphericity test; $\mathrm{P}<0.001)$.

The correlations of the total PDAQ-15 with the Lawton IADL scale and depression domain of HADS scores were 0.95 and -0.71 , respectively, indicating strong 
Table 1. Sample demographic and clinical characteristics

\begin{tabular}{|c|c|c|c|c|c|}
\hline \multirow{2}{*}{\multicolumn{2}{|c|}{ Variables }} & \multicolumn{4}{|c|}{ Mean $\pm S D / \%$} \\
\hline & & PD-NC (n=75) & PD-MCI $(n=50)$ & $\operatorname{PDD}(n=40)$ & Total $(n=165)$ \\
\hline \multicolumn{2}{|c|}{ Age (y) } & $59.50 \pm 9.75$ & $64.90 \pm 8.06$ & $70.82 \pm 8.82$ & $63.88 \pm 10.08$ \\
\hline \multicolumn{2}{|c|}{ Education (y) } & $10.89 \pm 4.81$ & $8.02 \pm 5.32$ & $6.42 \pm 5.85$ & $8.93 \pm 5.53$ \\
\hline Gender & Male & 70.7 & 64 & 55 & 64.8 \\
\hline \multicolumn{2}{|c|}{ Age of onset (y) } & $52.88 \pm 11.1$ & $57.18 \pm 10.13$ & $62.75 \pm 9.28$ & $56.57 \pm 11.09$ \\
\hline \multicolumn{2}{|c|}{ Disease duration (y) } & $6.65 \pm 5.21$ & $7.72 \pm 5.48$ & $8.02 \pm 5.53$ & $7.30 \pm 5.37$ \\
\hline \multicolumn{2}{|c|}{ LEDD (mg/day) } & $690 / 53 \pm 339.25$ & $706.37 \pm 466.70$ & $649.15 \pm 321.07$ & $685.30 \pm 376.81$ \\
\hline \multicolumn{2}{|c|}{ On DA } & 10.7 & 30 & 10 & 16.4 \\
\hline \multirow{5}{*}{$H \& Y$} & 1 & 53.3 & 12 & 2.5 & 28.5 \\
\hline & 2 & 30.7 & 44 & 32.5 & 35.2 \\
\hline & 3 & 10.7 & 24 & 12.5 & 15.2 \\
\hline & 4 & 0 & 0 & 27.5 & 6.7 \\
\hline & 5 & 5.3 & 20 & 25 & 14.5 \\
\hline \multicolumn{2}{|c|}{ HADS anxiety } & $7.25 \pm 5.29$ & $9.58 \pm 4.62$ & $15.40 \pm 4.93$ & $9.93 \pm 5.95$ \\
\hline \multicolumn{2}{|c|}{ HADS depression } & $5.53 \pm 4.41$ & $9.54 \pm 5.68$ & $16.55 \pm 5.37$ & $9.41 \pm 6.68$ \\
\hline \multicolumn{2}{|c|}{ Lawton IADL scale } & $7.74 \pm 0.43$ & $6.10 \pm 0.61$ & $2.82 \pm 2.06$ & $6.05 \pm 2.25$ \\
\hline
\end{tabular}

LEDD: total levodopa equivalent daily dose; DA: dopamine agonist; H\&Y: Hoehn \& Yahr; HADS: Hospital Anxiety and Depression Scale; IADL: instrumental activities of daily living.

convergent validity. The correlation between the total PDAQ-15 score and the anxiety domain of HADS was -0.66 , which indicates a moderate association.

PDAQ-15 scores for PD-NC, PD-MCI, and PDD are presented in Table 3. The Kruskal-Wallis test analysis showed a significant difference between PD cognitive stages for PDAQ-15 ( $\mathrm{P}<0.001)$. PDAQ-15 had a moderate-high effect size $(E S=0.62)$ in the separation of the PD-NC from PD-MCI, high effect size in separation of the PD-MCI from PDD (ES=0.88) and PD-NC from PDD (ES=0.93).

\section{Discussion}

The present study aimed to describe the psychometric properties of PDAQ-15. It should be noted that this questionnaire has not yet been validated in other cultures, so the initial PDAQ-15 validation study by the Brennan et al. was the only available study we had to compare to ours (Brennan et al., 2016a, 2016b).

We have found that PDAQ-15 is a valid and reliable instrument to assess cognitive IADL in PD patients. The ceiling effect was observed for this questionnaire, indicating the number of the highest score and better IADL function of patients. Most PD patients in our study were in the normal cognitive stage of the CDR classification, and this finding could be due to the combination of our sample. Nevertheless, the superiority of the PDAQ-15 was observed in its ability to identify a wide spectrum of functional decline correlated to cognitive impairments in PD.

The distribution of scores indicates which items are very easy or difficult in general. For example, item 9 and item 15 had the lowest and highest frequency of the highest score (4 points), respectively. 
Table 2. Inter-item correlation for the Penn Parkinson's Daily Activities Questionnaire-15 (PDAQ-15)

\begin{tabular}{|c|c|c|c|c|c|c|c|c|c|c|c|c|c|c|c|}
\hline \multirow{2}{*}{$\begin{array}{l}\text { Items of } \\
\text { PDAQ-15 }\end{array}$} & \multicolumn{15}{|c|}{ Inter Item Correlation Matrix } \\
\hline & 1 & 2 & 3 & 4 & 5 & 6 & 7 & 8 & 9 & 10 & 11 & 12 & 13 & 14 & 15 \\
\hline 1 & 1 & & & & & & & & & & & & & & \\
\hline 2 & 0.96 & 1 & & & & & & & & & & & & & \\
\hline 3 & 0.94 & 0.96 & 1 & & & & & & & & & & & & \\
\hline 4 & 0.95 & 0.93 & 0.92 & 1 & & & & & & & & & & & \\
\hline 5 & 0.95 & 0.96 & 0.98 & 0.93 & 1 & & & & & & & & & & \\
\hline 6 & 0.93 & 0.94 & 0.97 & 0.93 & 0.96 & 1 & & & & & & & & & \\
\hline 7 & 0.93 & 0.93 & 0.91 & 0.94 & 0.91 & 0.91 & 1 & & & & & & & & \\
\hline 8 & 0.92 & 0.90 & 0.92 & 0.94 & 0.91 & 0.93 & 0.95 & 1 & & & & & & & \\
\hline 9 & 0.92 & 0.91 & 0.90 & 0.93 & 0.91 & 0.90 & 0.97 & 0.95 & 1 & & & & & & \\
\hline 10 & 0.95 & 0.96 & 0.96 & 0.95 & 0.97 & 0.95 & 0.93 & 0.93 & 0.91 & 1 & & & & & \\
\hline 11 & 0.94 & 0.96 & 0.98 & 0.92 & 0.97 & 0.97 & 0.90 & 0.92 & 0.90 & 0.96 & 1 & & & & \\
\hline 12 & 0.95 & 0.93 & 0.93 & 0.97 & 0.94 & 0.94 & 0.95 & 0.94 & 0.93 & 0.95 & 0.93 & 1 & & & \\
\hline 13 & 0.95 & 0.95 & 0.94 & 0.94 & 0.95 & 0.95 & 0.95 & 0.92 & 0.93 & 0.94 & 0.94 & 0.96 & 1 & & \\
\hline 14 & 0.94 & 0.94 & 0.98 & 0.93 & 0.97 & 0.99 & 0.90 & 0.92 & 0.90 & 0.95 & 0.98 & 0.94 & 0.95 & 1 & \\
\hline 15 & 0.94 & 0.95 & 0.98 & 0.91 & 0.97 & 0.97 & 0.90 & 0.92 & 0.90 & 0.96 & 0.98 & 0.93 & 0.94 & 0.97 & 1 \\
\hline
\end{tabular}

1. Reading the newspaper or magazine, 2. Kepping track of time, 3. Counting the correct amount of money, 4. Reading and following complex instructions, 5. Handling an unfamiliar problem, 6. Explaining how to do something involving several steps to another person, 7. Remembering a list of 4 or 5 errands without writing it down, 8. Using a map to tell where to go, 9. Remembering new information like phone numbers or simple instructions, 10 . Doing more than one thing at a time, 11. Learning to use new gadgets or machines around the house, 12. Understanding his/her personal financial affairs, 13. Maintaining or completing a train of thought, 14. Discussing a TV show, book, movie or current events, 15 . Remebering what day and month it is.

Table 3. PDAQ-15 scores by cognitive diagnosis

\begin{tabular}{|c|c|c|c|c|c|c|}
\hline & \multirow{2}{*}{$\mathbf{N}$} & \multirow{2}{*}{ Mean \pm SD } & \multicolumn{2}{|c|}{$95 \% \mathrm{Cl}$ for mean } & \multirow{2}{*}{ Min } & \multirow{2}{*}{ Max } \\
\hline & & & Lower Bound & Upper Bound & & \\
\hline PD-NC & 75 & $56.78 \pm 4.73$ & 55.69 & 57.87 & 45 & 60 \\
\hline PD-MCI & 50 & $47.78 \pm 6.33$ & 45.97 & 49.58 & 27 & 53 \\
\hline PDD & 40 & $13.32 \pm 10.77$ & 9.87 & 16.77 & 0 & 30 \\
\hline Total & 165 & $43.52 \pm 18.92$ & 40.61 & 46.43 & 0 & 60 \\
\hline
\end{tabular}

PD-NC: Parkinson's disease patients with normal cognition; PD-MCI: Parkinson's disease patients with mild cognitive impairment; PDD: Parkinson's disease patients with dementia 
The present study showed high test-retest reliability indicating high homogeneity of respondent's scores in two separate situations, which is in line with the results of the study done by Brennan et al. (2016a).

The strong agreement for the inter-rater reliability and high Cronbach alpha and item total-correlation indicate that all items in the PDAQ-15 are comparable in correctly detecting functional impairment, similar to the results of the study by Brennan et al. (2016a, 2016b).

We found a high correlation between PDAQ-15 and Lawton IADL scale, which is in line with the studies were done by Brennan et al. (2016a, 2016b). Also, in this study, the correlation between PDAQ-15 and with depression domain of HADS was high. Our study showed that the PDAQ-15 could find PD patients with a decline in IADL function and depression.

The SEM value obtained in this study is supported by the finding by Brennan et al., which indicates that this questionnaire has an adequate precision (low measurement error) for PD patients with varying levels of IADL ability (Brennan et al., 2016a).

The unidimensionality of the PDAQ-15 indicates that all measurement items belong to a single concept (cognitive IADL), confirmed by the study conducted by Brennan et al. in the initial testing of PDAQ (Brennan et al., 2016a).

The results also showed that the PDAQ-15 could well discriminate between PD-NC, PD-MCI, and PDD based on their functional impairment, which is consistent with the results of Brennan et al. (2016a, 2016b). These statistically significant findings could be helpful for the interpretation of outcomes in clinical trials examining the impact of cognition on IADLs in the PD population.

However, there are some limitations to our study. Because the PDAQ-15 is a new questionnaire, we did not have other studies to compare with ours. Additionally, we did not have the Persian validation of PD-specific scales to assess cognition and IADL function. All evaluations were performed while in on-state, and results may have been impacted if PD patients were assessed in the off-state.

In summary, the PDAQ-15 appears to be a valid and reliable instrument to detect the wide spectrum of IADL functioning decline associated with cognitive impairment in PD among the Iranian population. It can be used as an appropriate tool for assessing cognitive IADL in $\mathrm{PD}$ in clinical and research settings.

A future study is needed to assess the ability of PDAQ15 to detect meaningful changes in cognition IADL function over time and relative to therapeutic interventions. Further investigation on the transcultural validation of PDAQ-15 is also warranted.

\section{Ethical Considerations}

\section{Compliance with ethical guidelines}

All ethical principles are considered in this article. The participants were informed of the purpose of the research and its implementation stages. They were also assured about the confidentiality of their information and were free to leave the study whenever they wished, and if desired, the research results would be available to them. A written consent has been obtained from the subjects. Principles of the Helsinki Convention was also observed.

\section{Funding}

The paper was extracted from a research project of Negar Nikbakht, Mehdi Rezaee, Minoo Kalantari, Seyed Mehdi Tabatabaee, Gholam-Ali Shahidi, Department of Occupational Therapy, Faculty of Rehabilitation, Shahid Behshti University of Medical Sciences.

\section{Authors' contributions}

Conceptualization and Supervision: Negar Nikbakht and Mehdi Rezaee and Minoo Kalantari; Methodology: Seyed Mehdi Tabatabaee; Investigation, Writing-original draft, and Writing-review \& editing: All authors; Data collection: Negar Nikbakht and Gholam-Ali Shahidi; Data analysis: Negar Nikbakht and Seyed Mehdi Tabatabaee

\section{Conflict of interest}

The authors declared no conflict of interest.

\section{Acknowledgments}

We thank the patients and their families for participating in this study. 


\section{References}

Auclair-Ouellet, N., Lieberman, P., \& Monchi, O. (2017). Contribution of language studies to the understanding of cognitive impairment and its progression over time in Parkinson's disease. Neuroscience $\mathcal{E}$ Biobehavioral Reviews, 80, 657-672. [PMID]

Brennan, L., Siderowf, A., Rubright, J. D., Rick, J., Dahodwala, N., \& Duda, J. E., et al. (2016a). Development and initial testing of the Penn Parkinson's Daily Activities Questionnaire. Movement Disorders: Official Journal of the Movement Disorder Society, 31(1), 126-134. [PMID] [PMCID]

Brennan, L., Siderowf, A., Rubright, J. D., Rick, J., Dahodwala, N., \& Duda, J. E., et al. (2016). The Penn Parkinson's Daily Activities Questionnaire-15: Psychometric properties of a brief assessment of cognitive instrumental activities of daily living in Parkinson's disease. Parkinsonism $\mathcal{E}$ Related Disorders, 25, 21-26. [PMID] [PMCID]

Cahn, D. A., Sullivan, E. V., Shear, P. K., Pfefferbaum, A., Heit, G., \& Silverberg, G. (1998). Differential contributions of cognitive and motor component processes to physical and instrumental activities of daily living in Parkinson's disease. Archives of Clinical Neuropsychology, 13(7), 575-583. [DOI:10.1093/arclin/13.7.575]

Christ, J. B., Fruhmann Berger, M., Riedl, E., Prakash, D., Csoti, I., \& Molt, W., et al. (2013). How precise are activities of daily living scales for the diagnosis of Parkinson's disease dementia? A pilot study. Parkinsonism $\mathcal{E}$ Related Disorders, 19(3), 371-374. [PMID]

Dancis, A., \& Cotter, V. T. (2015). Diagnosis and management of cognitive impairment in Parkinson's disease. The Journal for Nurse Practitioners, 11(3), 307-313. [DOI:10.1016/j.nurpra.2014.11.023]

Federico, A., Trentin, M., Zanette, G., Mapelli, D., Picelli, A., \& Smania, N., et al. (2017). Diagnosing mild cognitive impairment in Parkinson's disease: Which tests perform best in the Italian population? Neurological Sciences, 38(8), 1461-1468. [PMID]

Fernández-Bobadilla, R., Martínez-Horta, S., Marín-Lahoz, J., Horta-Barba, A., Pagonabarraga, J., \& Kulisevsky, J. (2017). Development and validation of an alternative version of the Parkinson's Disease-Cognitive Rating Scale (PD-CRS) Parkinsonism E Related Disorders, 43, 73-77. [PMID]

Foster, E. R., \& Hershey, T. (2011). Everyday executive function is associated with activity participation in Parkinson disease without dementia. OTJR: Occupation, Participation and Health, 31(1), 16-22. [PMCID]

Gorsuch, R. L. (1997). Exploratory factor analysis: Its role in item analysis. Journal of Personality Assessment, 68(3), 532-560. [PMID]

Graf, C. (2008). The Lawton instrumental activities of daily living scale. The American Journal of Nursing, 108(4), 52-62. [PMID]

Hays, R. D., Anderson, R., \& Revicki, D. (1993). Psychometric considerations in evaluating health-related quality of life measures. Quality of Life Research, 2(6), 441-449. [PMID]

Hughes, A. J., Daniel, S. E., Kilford, L., \& Lees, A. J. (1992) Accuracy of clinical diagnosis of idiopathic Parkinson's disease: A clinico-pathological study of 100 cases. Journal of Neurology, Neurosurgery \& Psychiatry, 55(3), 181-184. [PMID] [PMCID]
Husted, J. A., Cook, R. J., Farewell, V. T., \& Gladman, D. D. (2000). Methods for assessing responsiveness: A critical review and recommendations. Journal of Clinical Epidemiology, 53(5), 459-468. [DOI:10.1016/S0895-4356(99)00206-1]

Kulisevsky, J., Fernández de Bobadilla, R., Pagonabarraga, J., Martínez-Horta, S., Campolongo, A., \& García-Sánchez, C. et al. (2013). Measuring functional impact of cognitive impairment: Validation of the Parkinson's disease cognitive functional rating scale. Parkinsonism \& Related Disorders, 19(9), 812-817. [PMID]

Lawton, M., \& BRODY, E. M. (1970). Assessment of older people: Self-maintaining and instrumental activities of daily living. Nursing Research, 19(3), 278. [DOI:10.1097/00006199197005000-00029]

Leung, I. H., Walton, C. C., Hallock, H., Lewis, S. J., Valenzuela, M., \& Lampit, A. (2015). Cognitive training in Parkinson disease: A systematic review and meta-analysis. Neurology, 85(21), 1843-1851. [PMID] [PMCID]

Aaronson, N., Alonso, J., Burnam, A., Lohr, K. N., Patrick, D L., \& Perrin, E., et al. (2002). Assessing health status and quality-of-life instruments: attributes and review criteria. Quality of Life Research, 11(3), 193-205. [PMID]

Martin, R. C., Triebel, K. L., Kennedy, R. E., Nicholas, A. P. Watts, R. L., \& Stover, N. P., et al. (2013). Impaired financial abilities in Parkinson's disease patients with mild cognitive impairment and dementia. Parkinsonism \& Related Disorders, 19(11), 986-990. [PMID] [PMCID]

McHorney, C. A., \& Tarlov, A. R. (1995). Individual-patient monitoring in clinical practice: Are available health status surveys adequate? Quality of Life Research, 4(4), 293-307. [PMID]

Mehdizadeh, M., Martinez-Martin, P., Habibi, S. A., Nikbakht, N., Alvandi, F., \& Bazipoor, P., et al. (2019). The association of balance, fear of falling, and daily activities with drug phases and severity of disease in patients with parkinson. Basic and Clinical Neuroscience, 10(4), 355-362. [PMID] [PMCID]

Montazeri, A., Vahdaninia, M., Ebrahimi, M., \& Jarvandi, S (2003). The Hospital Anxiety and Depression Scale (HADS): Translation and validation study of the Iranian version. Health and Quality Of Life Outcomes, 1, 14. [PMID] [PMCID]

Morris, J. C. (1997). Clinical dementia rating: A reliable and valid diagnostic and staging measure for dementia of the Alzheimer type. International Psychogeriatrics, 9(S1), 173-176. [PMID]

Nikbakht, N., Rezaee, M., Kalantari, M., \& Tabatabaee, S. M. (2018). The Persian version of Penn Parkinson's Daily Activities Questionnaire-15: Face and content validity. Journal of Clinical Physiotherapy Research, 3(1), 5-8. [Link]

Piedmont, R. L. (2014). Inter-item correlations. In: A. C. Michalo (Ed), Encyclopedia of quality of life and well-being research (pp 3303-3304). Dordrecht: Springer. [DOI:10.1007/978-94007-0753-5_1493]

Rasovska, H., \& Rektorova, I. (2011). Instrumental activities of daily living in Parkinson's disease dementia as compared with Alzheimer's disease: Relationship to motor disability and cognitive deficits: A pilot study. Journal of the Neurological Sciences, 310(1-2), 279-282. [PMID] 
Ruzafa-Valiente, E., Fernández-Bobadilla, R., García-Sánchez, C., Pagonabarraga, J., Martínez-Horta, S., \& Kulisevsky, J. (2016). Parkinson's Disease-Cognitive Functional Rating Scale across different conditions and degrees of cognitive impairment. Journal of The Neurological Sciences, 361, 66-71. [PMID]

Siciliano, M., De Micco, R., Trojano, L., De Stefano, M., Baiano, C., \& Passaniti, C., et al. (2017). Cognitive impairment is associated with Hoehn and Yahr stages in early, de novo Parkinson disease patients. Parkinsonism \& Related Disorders, 41, 86-91. [PMID]

Simon, S. D. (2006). Statistical evidence in medical trials: what do the data really tell us? In: Baylor University Medical Center. [PMCID]

Hassani Mehraban, A., Soltanmohamadi, Y., Akbarfahimi, M., \& Taghizadeh, G. (2014). Validity and reliability of the Persian version of Lawton instrumental activities of daily living scale among patients with dementia. Medical Journal of the Islamic Republic of Iran, 28, 25. [PMID]

Streiner, D. L., Norman, G. R., \& Cairney, J. (2015). Health measurement scales: A practical guide to their development and use. Oxford: Oxford University Press. [DOI:10.1093/ med/9780199685219.001.0001]

Taghizadeh, G., Martinez-Martin, P., Fereshtehnejad, S. M., Habibi, S. A., Nikbakht, N., \& Alizadeh, N. H., et al. (2018). Psychometric properties of the Berg balance scale in idiopathic Parkinson' disease in the drug off-phase. Neurological Sciences, 39(12), 2175-2181. [PMID]

Terwee, C. B., Bot, S. D., de Boer, M. R., van der Windt, D. A., Knol, D. L., \& Dekker, J., et al. (2007). Quality criteria were proposed for measurement properties of health status questionnaires. Journal of Clinical Epidemiology, 60(1), 34-42. [PMID]

Wyman-Chick, K. A., \& Scott, B. J. (2015). Development of clinical dementia rating scale cut-off scores for patients with parkinson's disease. Movement Disorders Clinical Practice, 2(3), 243-248. [PMID]

Zigmond, A. S., \& Snaith, R. P. (1983). The hospital anxiety and depression scale. Acta Psychiatrica Scandinavica, 67(6), 361-370. [PMID] 
This Page Intentionally Left Blank 